ARTICLE ID PLACEHOLDER

\title{
Design and characteristics of a low-frequency magnetic probe for magnetic profile
} measurements at Wendelstein 7-X
A. Knieps, $\left.{ }^{1},{ }^{2}\right)$ Y. Liang,,${ }^{1,2}$ P. Drews, ${ }^{1}$ M. Endler, ${ }^{3}$ O. Grulke, ${ }^{3}$ Z. Huang,,${ }^{1,2}$ C. Killer, ${ }^{3}$ S. Liu, ${ }^{1,4}$ D. Nicolai, ${ }^{1}$ K. Rahbarnia, ${ }^{3}$ N. Sandri, ${ }^{1}$ G. Satheeswaran, ${ }^{1}$ and the Wendelstein $7-X$ team
1) Forschungszentrum Jülich GmbH, Institut für Energie- und Klimaforschung Plasmaphysik, Partner of the Trilateral Euregio Cluster (TEC), Jülich, 52425, Germany
2) International Joint Research Laboratory of Magnetic Confinement Fusion and Plasma Physics, State Key Laboratory of Advanced Electromagnetic Engineering and Technology, School of Electrical and Electronic Engineering, Huazhong University of Science and Technology, Wuhan, 430074,
People's Republic of China
3) Max-Planck-Institut für Plasmaphysik, Greifswald, 17491, Germany
4) Institute of Plasma Physics, Chinese Academy of Sciences, Hefei 230031, Peoples Republic of China

Equilibrium analysis in fusion-devices usually relies on plasma pressure profiles and magnetic measurements outside the plasma. The kinetic profiles can give indirect information about the equilibrium magnetic field, while the stationary magnetic diagnostics can not resolve current distributions on a smaller scale. This work presents a reciprocating magnetic probe, designed to provide direct plasma response measurements of the magnetic field in the scrape-off layer of Wendelstein 7-X. Hardware design and frequency characteristics are discussed, and a post-processing technique for extending the lower frequency cutoff of the integration scheme is presented.

\section{INTRODUCTION}

Low-frequency noise is a common hurdle in magnetic diagnostics based on integrated coil signals, due to the large relative am-

a)Electronic mail: a.knieps@fz-juelich.de plification of low-frequency components showing up as low-frequency drifts. The long discharge durations of the Wendelstein 7-X (W7-X) stellarator ${ }^{112}$ further emphasize this issue. Discharges frequently exceed $10 \mathrm{~s}$ in length and have reached up to $100 \mathrm{~s}$ 
already, with 30 min discharges planned for a future campaign. Signal chopping (either by zeroing or inversion of the input voltage) can be used to capture low-frequency drift in processing stages behind the chopper ${ }^{3 / 4}$, but the chopper itself must be set up carefully to avoid channel cross talk and biases introduced by the chopping circuit. The chopped signal must then be interpreted in software to compensate both chopping the determined drift. However, recently, a new implementation strategy for signal chopping has emerged. Multiple vendors now offer operational amplifiers with an integrated stabilization circuit. The signal is chopped in the amplifier and used to determine an offset correction for the amplifier. Such an operational amplifier can then be integrated into a conventional analog integrator circuit, offering the advantages of chopper-based stabilization while retaining the simplicity of the design. This paper discusses a magnetic probe using such an analog integrator designed to measure the change of the edge magnetic field of W7-X during plasma operation.

At the plasma edge, W7-X relies on a chain of magnetic islands - intersected by its divertor target plates - for heat- and particle exhaust ${ }^{5-7}$. For high-performance long-pulse operation, the modification of these islands both due to a change in the magneto-hydrodynamic (MHD) equilibrium and bootstrap currents needs to be modeled to ensure operational safety and proper screening of the plasma core from edge impurities. Equilibrium calculation codes, such as $\operatorname{VMEC}^{8}$ and HINT ${ }^{9}$ rely on pressure profiles as an input. Usually, these codes also have additional free parameters, such as (usually) the boundary shape in the case of VMEC and the pressure averaging length for HINT. In the past, the presence of these free parameters has shown to result in differing results between the codes, even outside the plasma $a^{10}$. For validation of numerical models, it is preferred to rely on diagnostic measurements which are unrelated to the model inputs. One good benchmark for equilibrium models is the change in the magnetic field, both in topology (which can be observed indirectly with edge profiling diagnostics) and magnetic signals.

Magnetic diagnostics at W7-X include flux loops ${ }^{11}$, Rogowski coils ${ }^{12[13}$ and Mirnov coils localised at the vesse ${ }^{14}$. Usually, the observations made by these diagnostics are either cross-section averaged or localized outside of the plasma. A key observation, however, would be the local magnetic field change inside the magnetic islands. Hall sensors make in principle an excellent can- 
didate for such low-frequency observations of the magnetic field, but sensor designs that can operate under the high heat fluxes experienced by reciprocating probes are still in development ${ }^{15}$. Magnetic coils are a common feature in reciprocating probes $\underline{16 \mid 17}$ but are mainly used for fluctuation studies 18 . Equilibrium profile reconstruction from coil signals is challenging since the magnetic field created by the plasma (which is in the order of $1 \mathrm{mT}$ to $10 \mathrm{mT}$ ) is dominated by the spatial variation of the vacuum magnetic field (which is about $0.1 \mathrm{~T}$ in the outboard mid-plane of W7-X), which requires a broad dynamic range while retaining high precision.

In contrast to Hall sensors, the signal of a magnetic probe must be integrated to obtain the plasma response. Methods for integration of magnetic coil signals can be mostly divided into two branches, analog and digital. Analog methods mostly use operational amplifierbased circuitry to obtain an integrated signal before sampling. Digital integration methods sample the un-integrated input signal and integrate the time trace numerically. Digital integration methods have the advantage of high dynamic range and ease of implementation, as well as the potential to modify the integration scheme later on, but can show susceptibility to low-frequency drift errors in the signal acquisition chain. This challenge can be addressed by introducing a signal chopper early into the measurement chain for drift calibration. Additionally, digital integration requires continuous data acquisition. Analog integrators can by placed early in the measurement chain, but require a trade-off between dynamic range, stability against parasitic decay and input impedance.

\section{SETUP OF THE DIAGNOSTIC}

\section{A. The combined probe}

The combined probe is a diagnostic system for the integrated measurement of a multitude of plasma parameter profiles. Its front surface features a triple probe for electron temperature and density measurements, two protruding floating potential pins, a pair of up- and downstream facing Mach probes for parallel flow measurements, a gas inlet, a tungsten sample for exposition and an experimental ion-sensitive probe ${ }^{19}$. The probe interior - including the magnetic coils - is shielded by a Boron Nitride cover. The probe is designed for the Multi-Purpose Manipulator $(\mathrm{MPM})^{20121}$, which is located in the $\phi=200.8^{\circ}, z=-17 \mathrm{~cm}$ line and can theoretically plunge up to $35 \mathrm{~cm}$ inwards from its parking position. Large heat-fluxes on the probe however usually prevent it from plunging into the confined plasma core. Magnetic 
plasma response measurements are restricted to the outboard side of the magnetic islands, due to interference between the pickup coil system and currents drawn via the Langmuir probes. This interference would manifest itself as a jump in the integrated voltage occuring simultaneously with short transient current bursts appearing on the Langmuir probe channels. To ensure that smaller variations of this interference - potentially not visible to the naked eye on the raw signals - do not accumulate in the integrated signal, the error analysis was designed to be sensitive to differences in the magnetic field measurement during the inward and outward motion.

\section{B. The magnetic sensor}

The magnetic probe is a $3 \mathrm{D}$ pickup probe consisting of three concentric mutually orthogonal coils. Each coil consists of 500 windings of a $0.1 \mathrm{~mm}$ copper wire with a Polyimide film (Kapton) insulation. The coils have a (calculated) effective area per winding of $1.342 \mathrm{~cm}^{2}, 1.464 \mathrm{~cm}^{2}$ and $1.83 \mathrm{~cm}^{2}$ in radial, toroidal and vertical direction respectively. The common center point is offset $39.2 \mathrm{~mm}$ from the front-most pin, $111 \mathrm{~mm}$ from the base of the probe along the probe path, and $6 \mathrm{~mm}$ below the MPM center axis (see figure 1). For measured total effective areas, see table I. The design also features a differential coil pair (2198 windings each, oppositely wound, $1 \mathrm{~cm}$ apart) for gradient fluctuation measurements, but these coils were out of operation during the 2018 campaign. The pickup probe is connected via $14 \mathrm{~m}$ long twisted cable pairs to the integration circuit.

\section{Integration circuitry}

For this probe, an analog integrator was chosen over a chopper-stabilized digital integration for two reasons:

- Robustness of the integration scheme at limited sample rate and piece-wise signal acquisition

- Availability of high-performance stock components and ease of implementation (limited time-frame between the 2017 and 2018 experimental campaigns)

To minimize distortion of the signal prior to integration (mainly by $\frac{1}{f}$ noise), the integrator was implemented as an integrating pre-amplifier. It uses a standard inverting amplifier circuit with the parameters $R_{\mathrm{amp}}=10 \mathrm{k} \Omega$ and $C_{\mathrm{amp}}=10 \mu \mathrm{F}$ (see figure 2. In an ideal scenario, such an integrator has an amplification factor of $\alpha=$ $-10 \mathrm{~s}^{-1}$. The chosen operational amplifier AD8628 suppresses common-mode drift using a built-in signal chopper and feedback 


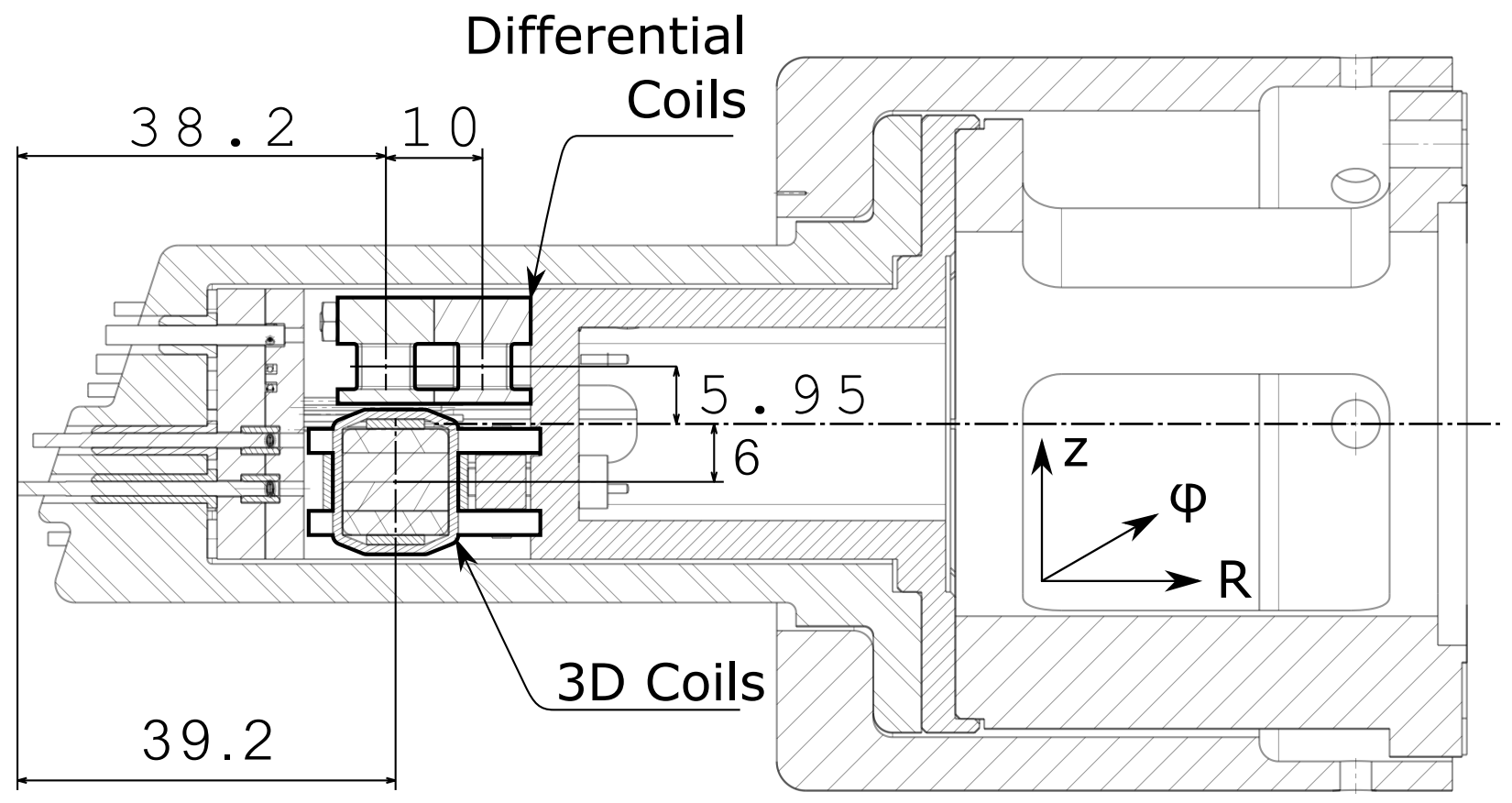

Figure 1. Location of the $3 \mathrm{D}$ and the differential coil sensors inside the combined probe

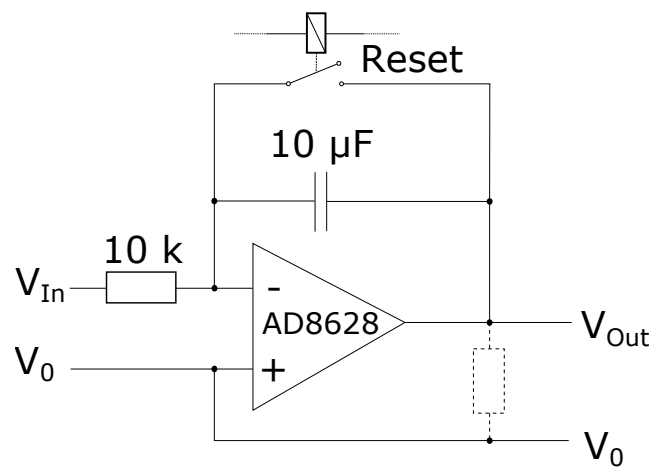

Figure 2. Simplified schematic of the integrating pre-amplifier (unintentional parasitic resistance displayed in dashed lines)

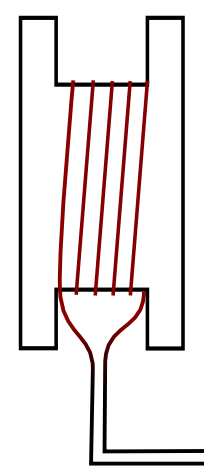

Figure 3. Setup of the signal processing stack for the pickup coil

\section{Signal acquisition}

loop. This configuration provides a strong noise reduction at low frequencies $(<10 \mathrm{~Hz})$ at the cost of additional output noise at the chopping frequency. This drawback is mitigated by pseudo-random chopping, spreading the noise up to $15 \mathrm{kHz}$ wide.

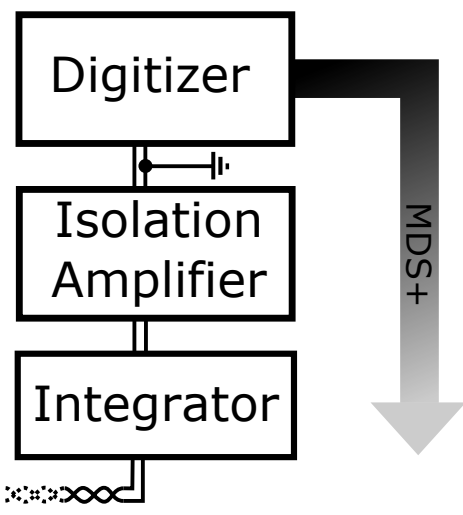

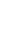




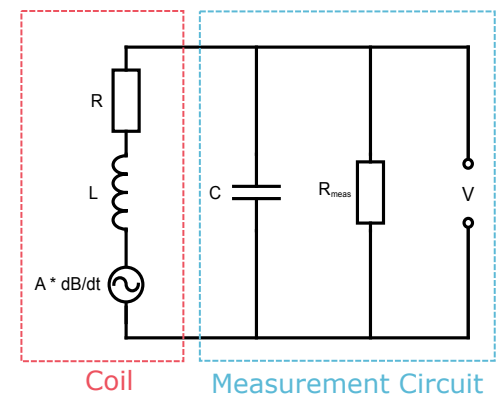

Figure 4. Circuitry model for the coil characterization. $\mathrm{A}, \mathrm{R}$ and $\mathrm{L}$ are the parameters to be determined, while $\mathrm{C}$ is externally set and $R_{\text {meas }}$ is a function of the voltage measurement device

amplifier before being recorded by a DTAQ ${ }^{\circledR}$ ACQ132CPCI and stored in an MDSplus 22|23 tree (see fig. 3). Data can be acquired in a single block or over multiple segments. The isolation amplifiers have a bandwidth of $2 \mathrm{MHz}$, while the sampling rate of the digitizers could be selected from a range between $250 \mathrm{kHz}$ (for up to $16 \mathrm{~s}$ ) and $2 \mathrm{MHz}$ (for up to $2 \mathrm{~s})$ during the last experimental campaign.

\section{COMPONENT}

\section{CHARACTERISTICS}

\section{A. Characteristics of the pickup coils}

The response curves of the pickup coil (phase and sensitivity) were determined in a pair of Helmholtz coils. The probe was set up as it would be inside the manipulator, so the measurement also includes shielding effects from the cover and metal com- ponents. Adding capacitors parallel to the voltage measurement allowed measurement of the coil's self-inductance and internal resistance. The equivalent circuitry for this measurement is shown in figure 4 and can be used to obtain the frequency response (equation 1) to an external magnetic field (substituting $\left.X=\widetilde{X} e^{i \omega t}\right)$, the derivation of which can be found in appendix A.

$$
\begin{aligned}
\frac{\widetilde{V}_{\text {coil }}}{\tilde{B}}= & \frac{i \omega A_{\text {coil }}}{h(\omega)} \\
h(\omega)= & 1+\frac{R_{\text {coil }}}{R_{\text {meas }}}-\omega^{2} L_{\text {coil }} C_{\text {meas }} \\
& +i \omega\left(R_{\text {coil }} C_{\text {meas }}+\frac{L_{\text {coil }}}{R_{\text {meas }}}\right)
\end{aligned}
$$

The parameter $C_{\text {meas }}$ was varied to better characterize the self-inductances, while the input impedance $R_{\text {meas }}$ of the measurement digitizer is fixed at $1 \mathrm{M} \Omega$. The parameters in table I were estimated by fitting equation 1 to the measurements in figure 5 in a range from $1 \mathrm{~Hz}$ to $10 \mathrm{kHz}$. In this range, the characteristics of the signal cables do not yet play a significant role. If one were doing a characterization into the $\mathrm{MHz}$ regime, one would have to take into account transmission delays as well as impedance matching effects. The nonmonotonic relation between the effective area $A$ and the inductance $L$ shows that the exact winding geometry of the coil plays an important role in determining the self-inductance. Given that the dynamic ranges have approximately a $2: 1: 1$ ratio for toroidal, vertical 

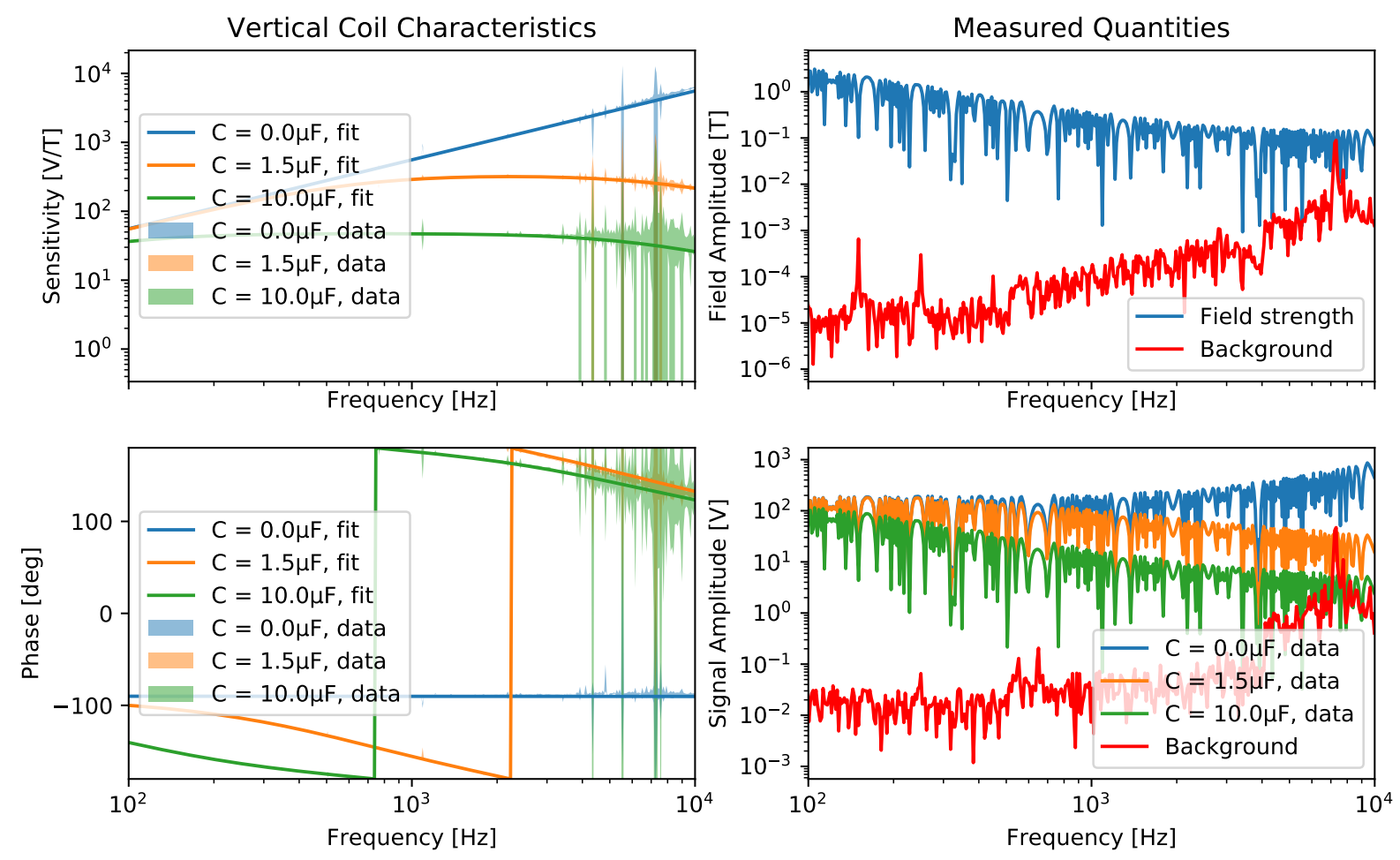

Figure 5. Input and output measurements - as well as background levels - for the radial sensitivity measurement

\begin{tabular}{|c|c|c|c|c|c|}
\hline Coil & $A_{\text {radial }}\left[\mathrm{cm}^{2}\right]$ & $A_{\text {toroidal }}\left[\mathrm{cm}^{2}\right]$ & $A_{\text {vertical }}\left[\mathrm{cm}^{2}\right]$ & $L[\mathrm{mH}]$ & $R[\Omega]$ \\
\hline \hline Radial & $675 \pm 10$ & $3.9_{-3.9}^{+5}$ & $6.9 \pm 5$ & $3.0 \pm 0.6$ & $167 \pm 10$ \\
\hline Toroidal & $1.9_{-1.9}^{+5}$ & $707 \pm 4$ & $5.4 \pm 5$ & $2.7 \pm 0.1$ & $167 \pm 10$ \\
\hline Vertical & $10.7 \pm 5$ & $3.0_{-3}^{+5}$ & $888 \pm 5$ & $3.4 \pm 0.1$ & $187 \pm 2$ \\
\hline
\end{tabular}

Table I. Empirically obtained coil parameters

\begin{tabular}{|c|c|c|}
\hline Coil 1 & Coil 2 & Mutual inductance $[\mu \mathrm{H}]$ \\
\hline \hline Toroidal & Radial & 34 \\
\hline Vertical & Radial & 18 \\
\hline Toroidal & Vertical & 16 \\
\hline
\end{tabular}

Table II. Upper bounds for mutual inductances of the coils

and radial signals respectively, the additional error from cross-direction coupling should be bounded at about $2 \%$, which is far below the intrinsic error level of the measurement itself (see section $\mathrm{V}$ for a discussion of the magnetic profile error). This limit of course only holds as long as the integration circuitry operates 
linearly, as this implies proper error cancellation when combining field measurements.

The uncertainties of the measurement in the upper-frequency region are related to two effects, which are visible in figure 5. Firstly, the current amplitude from the KEPCO power supply powering the Helmholtz coils drops (as the current supply is driven near its specified frequency limit of $10 \mathrm{kHz}$ ), reducing both the magnetic field and the coil signal. Secondly, there is an increase in background noise near $8 \mathrm{kHz}$, which causes the signal level to drop below the uncertainty. The noise peak obtained by the background characterization (where the power supply was set to a zero control voltage) exceeds the measured signal in magnitude at a few frequencies in the coil voltage measurement. This indicates a noise reduction when the power supply is actively driven to a specific frequency, which is possible if the power supply contains a nonlinear feedback loop. This means that the background measurement is likely an overestimation of the actual measurement error, but obtaining a more accurate estimate is experimentally challenging.

By replacing the external Helmholtz coil pair with the pick-up coils themselves as the magnetic field sources, the mutual inductances of the sensors can be determined. Due to the high ohmic resistivity of the coil, only small source currents could be applied. Up- per bound measurements obtained as such are shown in table [II. Due to the mutual inductances being two orders or magnitude below the self-inductances, and the similar dynamic range of the signals, we assume inductive effects to be dominated by the selfinductances.

\section{B. Characteristics of the analog integrator}

In a simple linear model the integrator can be described using equation 2 with the (integrating) input amplification $\alpha$ and the output signal decay time $\tau$.

$$
\frac{d V_{\text {out }}}{d t}=\alpha V_{\text {coil }}-\tau^{-1} V_{\text {out }}
$$

This model implies the transfer function $\widetilde{V}_{\text {out }}=\frac{\alpha}{\tau^{-1}+i \omega} \widetilde{V}_{\text {coil }}$ which has a $1 / \sqrt{2}$ cutoff at $f_{1 / \sqrt{2}}=\frac{1}{2 \pi \tau}$. Table III shows empirical parameters for model 2, obtained by fitting it onto the response to a single sine-shaped pulse of $500 \mathrm{~ms}$ duration mimicking a typical magnetic signal during a manipulator plunge. The high decay rate $\tau^{-1}$ was found to coincide with $\mathrm{a} \approx 3 \mathrm{M} \Omega$ scale parasitic resistance, which was only present when the integrators were mounted on their auxiliary power supply board. This parasitic decay stresses the importance of proper isolation between the signal line and external circuitry and will be addressed in the next iteration of the cir- 


\begin{tabular}{|c|c|c|c|}
\hline Integrator & $\alpha\left[\mathrm{s}^{-1}\right]$ & $\tau^{-1}\left[\mathrm{~s}^{-1}\right]$ & $f_{1 / \sqrt{2}}[\mathrm{~Hz}]$ \\
\hline \hline Radial & -11.68 & 0.22 & 0.035 \\
\hline Toroidal & -11.42 & 0.31 & 0.049 \\
\hline Vertical & -11.34 & 0.35 & 0.055 \\
\hline \hline Ideal & -10 & 0 & 0 \\
\hline
\end{tabular}

Table III. Parameters for the linear integrator model described in equation 2

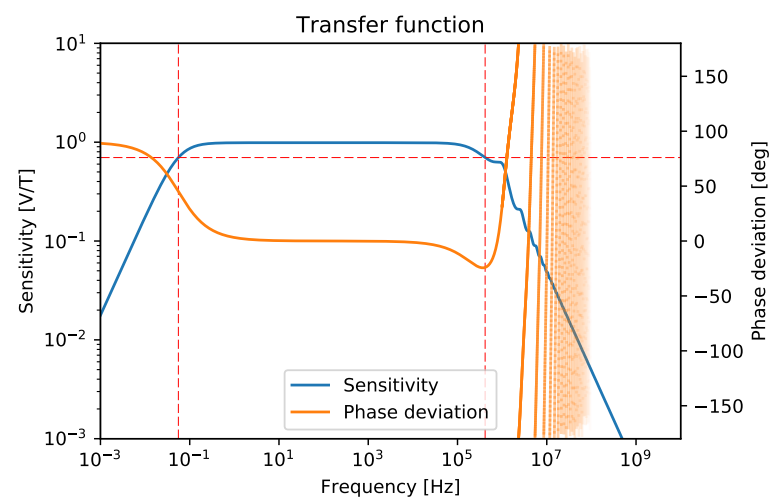

Figure 6. Total system characteristics of the different pickup coil systems (calculated using equation 3). Red lines mark the $1 / \sqrt{2}$ cutoff. To improve readability the phase axis does not include the $180^{\circ}$ from the inversion during integration.

cuitry.

IV. SYSTEM CHARACTERISTICS AND POST-PROCESSING

Combining the transfer function of the coil with the transfer function of the integrator leads to the total system transfer function de- rived in appendix $\mathrm{A}$.

$$
\begin{aligned}
\frac{\widetilde{V}_{\text {out }}}{\widetilde{B}} & =\frac{2 i \omega A \exp (i k l)}{(1-\lambda)(1-\kappa)+(1+\kappa)(\lambda+\exp (2 i k l))} \\
& \cdot \frac{\alpha}{1+\frac{1}{i \omega \tau}} \\
\lambda & =Z^{-1}\left(R_{\text {coil }}+i \omega L_{\text {coil }}\right) \\
\kappa & =Z\left(R_{\text {meas }}^{-1}+i \omega C_{\text {meas }}\right) \\
Z & =\sqrt{\frac{L_{\text {cable }}}{C_{\text {cable }}}-\frac{i}{\omega} \frac{R_{\text {cable }}}{C_{\text {cable }}}} \\
k & =\sqrt{\omega^{2} \frac{L_{\text {cable }} C_{\text {cable }}}{l^{2}}-i \omega \frac{R_{\text {cable }} C_{\text {cable }}}{l^{2}}}
\end{aligned}
$$

with the manipulator cable resistance $R_{\text {cable }}=(2.3 \pm 0.1) \Omega$, the cable capacitance $C_{\text {cable }}=(11 \pm 1) \mathrm{nF}$, its inductance $L_{\text {cable }}=$ $(9.4 \pm 0.1) \mu \mathrm{H}$ and the integrator's input impedance $R_{\text {meas }}=10 \mathrm{k} \Omega$. The length of the cable is implicitly captured in $R, C$ and $L$ and does not explicitly appear in either $k l$, $\kappa$ or $\lambda$. As can be seen in figure 6, the system has good sensitivity up into the low $\mathrm{kHz}$ regime, which is more than sufficient for accurate integration of the magnetic field in the absence of external disturbance. The lowfrequency behavior is, however, slightly problematic since the average measurement duration lies around 8-10 seconds. While the system is still sensitive at these frequencies, the deviations from the ideal case do already distort the magnetic field measurements in the form of a hysteresis opposite to the measured magnetic field change. Figure $7 \mathrm{~b}$ shows such a hysteresis in form of a negative offset after the plunge. 
To recover the correct field, two corrections are applied to the integrated signal:

- A linear compensation is added so that $V\left(t_{\text {start }}\right)=V\left(t_{\text {end }}\right)=0$

- A corrected integrated voltage is defined as $V_{\text {out,corr }}=\int_{t_{\text {start }}}^{t} V_{\text {coil }}$, estimating $V_{\text {coil }}$ using equation 2 . The backwards application of equation 2 results in a correction shown in equation 4.

$$
V_{\text {out }, \text { corr }}=\int_{t_{\text {start }}}^{t} \alpha^{-1}\left(\frac{d V_{\text {out }}}{d t}-\tau^{-1} V_{\text {out }}\right)
$$

It may seem unintuitive to first put in the effort to introduce an analog integrator, only to differentiate the signal and then again apply numerical integration. However, this scheme retains the low susceptibility against intermediate drifts in the signal processing chain. Additionally, $V_{\text {out }}$ only varies slowly between manipulator plunges (which does not apply for $\left.V_{\text {in }}\right)$, and therefore can be accurately interpolated if the signal acquisition is disabled in-between. A further advantage is that the $V_{\text {out }}$ component can be natively sampled at a low rate, while the unintegrated signal must either be sampled at a high rate or preprocessed using an analog low-pass filter. On the downside, while this correction scheme can be used to re-adjust frequency components of the order of $\tau^{-1}$, at even lower frequencies it exceedingly amplifies small noise components, requiring an improvement of the integrator hardware's $\tau$ value for long-pulse operation.

An example of the signal correction is shown in figure $7 \mathrm{~b}$. Besides a $10 \%$ reduction in peak height from $0.21 \mathrm{~T}$ to $0.19 \mathrm{~T}$ due to the correction of $\alpha$ from -10 to about -11 , the correction also significantly reduces the short-term offset of the magnetic field after the plunge, and brings the time-trace more in line with the position measurement (compare figures $7 \mathrm{a}$ and $\mathrm{b}$ ).

\section{MAGNETIC PROFILES}

As can be seen in figure $7 \mathrm{~b}-\mathrm{d}$, the postcorrected integrator output (also referred to as "hardware integrated" in figure 7 c and d) shows a significantly improved stability against low-frequency drifts, compared to both a simple software integration with a linear drift compensation (referred to as "software integrated" in figure 7 c and d) and the uncorrected integrator signal shown in figure 7 b. An unusual feature in the signal is a post-plunge oscillation at about $6 \mathrm{~Hz}$ that decays over about $1 \mathrm{~s}$. This feature is caused by a small vertical oscillation of the manipulator arm after the plunge, which is also visible in the arm's acceleration sensor (figure 7 e, see also ${ }^{20}$ ) after the plunge. The oscillation is not visible in all field components (compare figure $7 \mathrm{c}$ and $\mathrm{d}$ ). For 


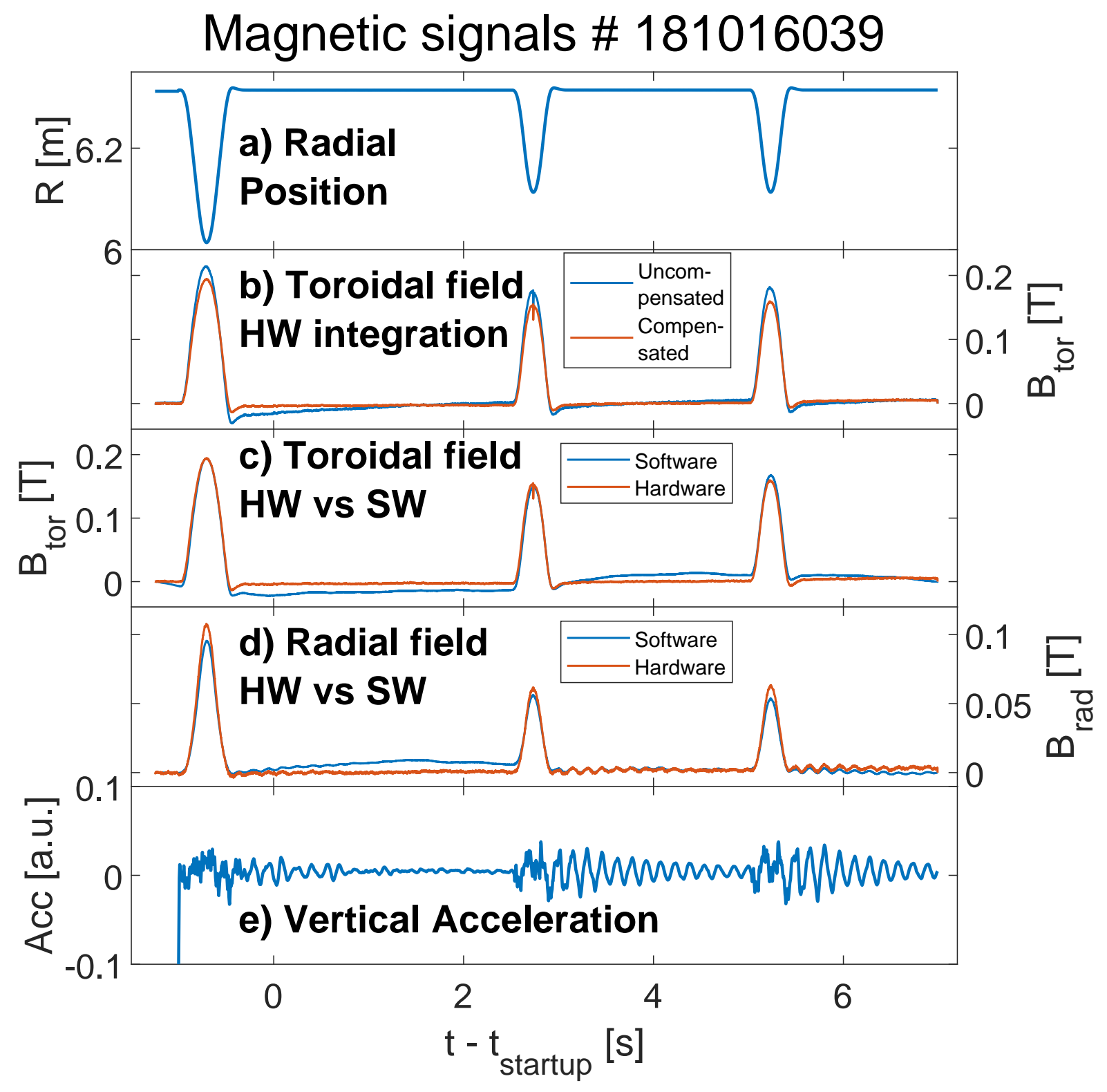

Figure 7. Time-traces of signals related to the magnetic probe measurement - a): Radial position of the magnetic probe, b): Comparison of hardware-integrated toroidal coil signal with and without post-compensation, c) Post-compensated hardware-integrated and software-integrated toroidal coil signal, d) Post-compensated hardware-integrated and software-integrated toroidal coil signal, e) Vertical acceleration measurement in the probe interface on the manipulator arm 

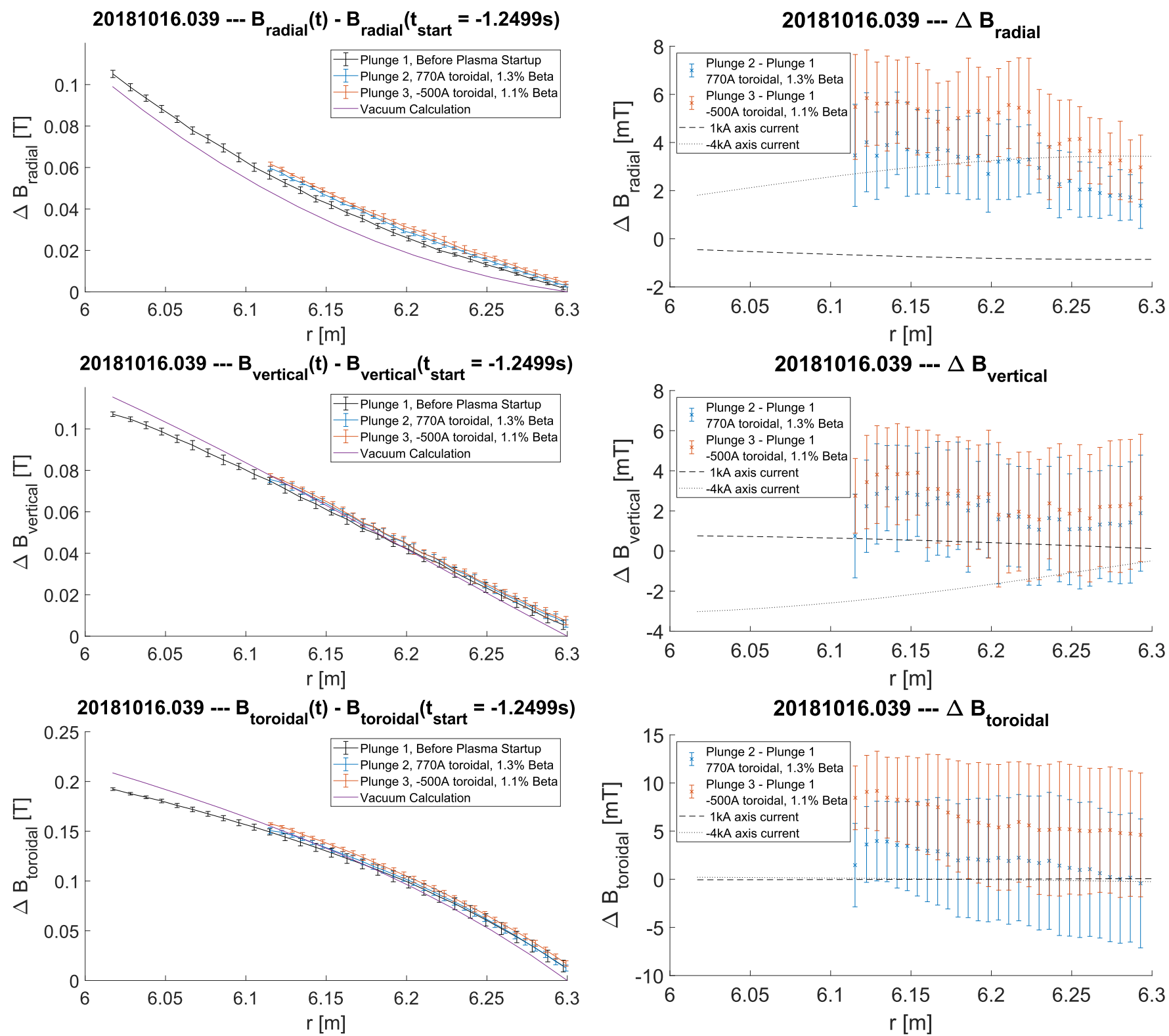

Figure 8. Measurements of magnetic field profiles (left) and plasma response (right)

profile derivation, the radial probe position the parking position. This overshoot is also is obtained using a laser-based distance mea- visible in the magnetic field measurement , surement system. As visible in figure 7 a, especially for the toroidal field profile (figure the manipulator arm does not immediately $7 \mathrm{~b}$ and $\mathrm{c}$ ), which has a steeper gradient at retract to the starting position, but instead the edge.

overshoots a little bit on the way back (likely due to inertia of the arm) before returning to

Figure 8 shows example radial profiles of 
all three magnetic field components, as well as theoretical field profile calculations obtained by applying the Biot-Savart law to the ideal CAD coil geometry. The radial profiles were obtained by binning the magnetic field time trace radially over the position signal, and using mean and standard deviation of the bin ensembles as value and error estimate respectively. This ensures that any differences between the inward and outward movement of the manipulator are appropriately captured in the error bars. To remove the influence of high-frequency MHD events, the magnetic signal was low-pass filtered before bin analysis. The cutoff frequency was varied between $50 \mathrm{~Hz}$ and $500 \mathrm{~Hz}$ without any significant variation of the profile or its errors. Toroidal current measurements were obtained from the W7-X Rogowski coil system, and peak plasma beta was estimated from Thomson scattering measurements for electron-density and -temperature measurements assuming $n_{I}=n_{e}$ for the ion density and $T_{i}=\min \left(T_{e}, 1.5 \mathrm{keV}\right)$ for the ion temperature. Because the profiles are obtained by integration, they are only defined up to a constant. This constant is chosen so that the integrated signal is $0 \mathrm{~T}$ at the start of the measurement (which is before the the $t=0$ s time of plasma startup).

The measured profiles do not agree perfectly with the expected profiles from a Biot-
Savart calculation assuming an ideal coil configuration. The first visible disagreement is a small positive offset from zero at the outer-most radius (which is the start- and end-point of the plunge) in all components. This is probably a small low-frequency deviation that could not be fully corrected during the post-processing. The additional slope disagreements in vertical and toroidal direction could be error fields related to the flattening of the main coils generating the magnetic field ${ }^{24}$. All of these deviations are not limited to this discharge, but can be observed systematically during the whole day in standard magnetic configuration.

Since the first probe plunge was always performed before plasma startup, it can be used as an experimental vacuum reference for plasma response calculation. Error analysis estimates that the magnetic probe has a field resolution of about $4 \mathrm{mT}$ in vertical and radial, and about $10 \mathrm{mT}$ in toroidal direction (the difference is likely due to the stronger overall magnetic field change in toroidal direction). The radial measurement in particular is accurate enough to detect a significant (given the measurement uncertainties) deviation between the vacuum magnetic field and the magnetic field during the discharge. When comparing the measured magnetic field change to the magnetic field gen- 
erated by a hypothetical current filament of $1 \mathrm{kA}$ on the magnetic axis, it can be seen, that an on-axis current of about $-4 \mathrm{kA}$ would be required to even poorly match the change in edge magnetic field. Given that the total toroidal current (as measured by the Rogowski coi ${ }^{133}$ ) is far lower than that, this magnetic field change is most likely caused by pressure-gradient-driven edge currents.

\section{SUMMARY}

A coil system for magnetic profile measurements using the Multi-PurposeManipulator was successfully employed at Wendelstein 7-X. Characterization of the probe and the integrator showed an upperfrequency $1 / \sqrt{2}$ cutoff of about $100 \mathrm{kHz}$ and a lower cutoff of $50 \mathrm{mHz}$. The lower cutoff could be extended using a post-processing technique. The resulting profiles are accurate enough to detect the plasma response in a $10 \mathrm{~s}$ discharge. While the recovery of the profile using post-processing (which amplifies any present low-frequency noise) is not ideal, we expect to reduce parasitic decay by multiple orders of magnitude in the next iteration of the design. Since the integrated signal good drift shows resilience under these aggravated conditions, we expect the stability to carry over into the next revision.

\section{ACKNOWLEDGEMENTS}

This work has been carried out within the framework of the EUROfusion Consortium and has received funding from the Euratom research and training programme 2014-2018 and 2019-2020 under grant agreement No 633053. The views and opinions expressed herein do not necessarily reflect those of the European Commission.

\section{DATA AVAILABILITY}

The data that support the findings of this study related to probe characterization are available from the corresponding author upon reasonable request. Raw data for the magnetic profile measurements were generated at the Wendelstein 7-X large scale facility. Derived data supporting the findings of this study are available from the corresponding author upon reasonable request.

\section{REFERENCES}

${ }^{1}$ H.-S Bosch, V. Erckmann, Ralf König, Felix Schauer, Reinhold Stadler, and A. Werner. Construction of wendelstein 7-x - engineering a steady-state stellarator. Plasma Science, IEEE Transactions on, 38:265 - 273, 04 2010. doi:10.1109/TPS.2009.2036918.

${ }^{2}$ Thomas Sunn Pedersen, Ralf König, Maciej Krychowiak, Marcin Jakubowski, JÃCEr- 
gen Baldzuhn, Sergey Bozhenkov, Golo Fuchert, Andreas Langenberg, Holger Niemann, Daihong Zhang, Kian Rahbarnia, Hans-Stephan Bosch, Yevgen Kazakov, Sebastijan Brezinsek, Yu Gao, and Novimir Pablant and. First results from divertor operation in wendelstein 7-x. Plasma Physics and Controlled Fusion, 61 (1):014035, nov 2018. doi:10.1088/13616587/aaec25. URL https://doi.org/10. 1088\%2F1361-6587\%2Faaec25.

${ }^{3}$ Andreas Werner. W7-x magnetic diagnostics: Performance of the digital integrator. Review of Scientific Instruments, 77(10): 10E307, 2006. doi:10.1063/1.2220073. URL https://doi.org/10.1063/1.2220073.

${ }^{4}$ Hans Hartfuss, R König, and A Werner. Diagnostics for steady state plasmas. Plasma Physics and Controlled Fusion, 48:R83, 09 2006. doi:10.1088/0741-3335/48/10/R01.

${ }^{5}$ F. Sardei, Y. Feng, P. Grigull, G. Herre, D. Hildebrandt, J. V. Hofmann, J. Kisslinger, R. Brakel, J. Das, J. Geiger, O. Heinrich, G. Kühner, H. Niedermeyer, D. Reiter, M. Richter-Glötzl, A. Runov, R. Schneider, U. Stroth, H. Verbeek, F. Wagner, R. Wolf, W7-AS Team, and NBI Group. Island divertor studies on W7AS. 241-243:135-148, 1997. Proceedings of the Twelveth International Conference on Plasma-Surface Interactions in Controlled Fusion Devices, Saint-Raphaël, France,
20-24 May 1996.

${ }^{6}$ R. König, P. Grigull, K. McCormick, Y. Feng, J. Kisslinger, A. Komori, S. Masuzaki, K. Matsuoka, T. Obiki, N. Ohyabu, H. Renner, F. Sardei, F. Wagner, and A. Werner. The divertor program in stellarators. 44 (11):2365-2422, November 2002. http://stacks.iop.org/PPCF/44/2365.

${ }^{7}$ Y. Feng, M. Kobayashi, T. Lunt, and D. Reiter. Comparison between stellarator and tokamak divertor transport. 53 (2):024009, February 2011. Special section on physics at the stellarator-tokamak interface, http://stacks.iop.org/PPCF/53/ 024009 .

${ }^{8}$ S. P. Hirshman. Steepest-descent moment method for three-dimensional magnetohydrodynamic equilibria. Physics of Fluids, 26(12):3553, 1983. doi:10.1063/1.864116.

${ }^{9}$ Yasuhiro Suzuki. HINT modeling of threedimensional tokamaks with resonant magnetic perturbation. Plasma Physics and Controlled Fusion, 59(5):054008, mar 2017. doi:10.1088/1361-6587/aa5adc.

${ }^{10} \mathrm{~S}$ A Lazerson, S Sakakibara, and Y Suzuki. A magnetic diagnostic code for $3 \mathrm{~d}$ fusion equilibria. Plasma Physics and Controlled Fusion, 55(2):025014, jan 2013. doi:10.1088/0741-3335/55/2/025014.

URL https://doi.org/10.1088\%

$2 \mathrm{~F} 0741-3335 \% 2 \mathrm{~F} 55 \% 2 \mathrm{~F} 2 \% 2 \mathrm{~F} 025014$. 
${ }^{11}$ K. Rahbarnia, H. Thomsen, U. Neuner, J. Schilling, J. Geiger, G. Fuchert, T. Andreeva, M. Endler, D. Hathiramani, T. Bluhm, M. Zilker, B.B. Carvalho, A. Werner, and Wendelstein 7-X team. Diamagnetic energy measurement during the first operational phase at the Wendelstein 7-X stellarator. Nuclear Fusion, 58(9):096010, jul 2018. doi:10.1088/17414326/aacab0.

${ }^{12}$ Andreas Werner, Michael Endler, Joachim Geiger, and Ralf Koenig. W7-X magnetic diagnostics: Rogowski coil performance for very long pulses. Review of Scientific Instruments, 79(10):10F122, oct 2008. doi: $10.1063 / 1.2957933$.

${ }^{13}$ M. Endler, B. Brucker, V. Bykov, A. Cardella, A. Carls, F. Dobmeier, A. Dudek, J. Fellinger, J. Geiger, K. Grosser, O. Grulke, D. Hartmann, D. Hathiramani, K. Höchel, M. Köppen, R. Laube, U. Neuner, X. Peng, K. Rahbarnia, K. Rummel, T. Sieber, S. Thiel, A. Vorköper, A. Werner, T. Windisch, and M.Y. Ye. Engineering design for the magnetic diagnostics of Wendelstein 7-X. Fusion Engineering and Design, 100:468-494, nov 2015. doi:10.1016/j.fusengdes.2015.07.020.

${ }^{14}$ M.Y. Ye, X.B. Peng, L. Fransico, and M. Endler. Thermal analysis of the Mirnov coils of Wendelstein 7-X. Fusion Engineer- ing and Design, 88(9-10):1844-1847, oct 2013. doi:10.1016/j.fusengdes.2013.05.087. ${ }^{15}$ S. Entler, I. Duran, M. Kocan, G. Vayakis, M. Kohout, J. Sebek, P. Sladek, O. Grover, and K. Vyborny. Prospects for the steady-state magnetic diagnostic based on antimony Hall sensors for future fusion power reactors. Fusion Engineering and Design, 146:526-530, sep 2019. doi: 10.1016/j.fusengdes.2019.01.013.

${ }^{16}$ Piero Agostinetti, Monica Spolaore, Matteo Brombin, Vannino Cervaro, Luca Franchin, Olaf Grulke, Carsten Killer, Emilio Martines, Maurizio Moresco, Simone Peruzzo, Nicola Vianello, and Michele Visentin. Design of a high resolution probe head for electromagnetic turbulence investigations in W7X. IEEE Transactions on Plasma Science, 46(5):1306-1311, may 2018. doi: $10.1109 /$ tps.2018.2799638

${ }^{17}$ P. Drews, Y. Liang, S. Liu, A. KrämerFlecken, O. Neubauer, J. Geiger, M. Rack, D. Nicolai, O. Grulke, C. Killer, N. Wang, A. Charl, B. Schweer, P. Denner, M. Henkel, Y. Gao, K. Hollfeld, G. Satheeswaran, N. Sandri, and D. Höschen. Measurement of the plasma edge profiles using the combined probe on W7-X. Nuclear Fusion, 57(12):126020, sep 2017. doi:10.1088/1741-4326/aa8385. 
${ }^{18}$ Christian M. Franck, Olaf Grulke, and Thomas Klinger. Magnetic fluctuation probe design and capacitive pickup rejection. Review of Scientific Instruments, 73(11):3768-3771, $2002 . \quad$ doi: 10.1063/1.1512341. URL https://doi. org $/ 10.1063 / 1.1512341$

${ }^{19}$ P Drews, C. Killer, J. Cosfeld, A. Knieps, S. Brezinsek, M. Jakubowski, C. Brandt, S. Bozhenkov, A. Dinklage, J. Cai, M. Endler, K. Hammond, M. Henkel, Y. Gao, J. Geiger, O. Grulke, D. Höschen, R. König, A. Krämer-Flecken, Y. Liang, Y. Li, S. Liu, H. Niemann, D. Nicolai, O. Neubauer, U. Neuner, M. Rack, K. Rahbarnia, L. Rudischhauser, N. Sandri, G. Satheeswaran, S. Schilling, H. Thomsen, T. Windisch, and S. Sereda. Edge plasma measurements on the OP $1.2 \mathrm{a}$ divertor plasmas at W7-X using the combined probe. Nuclear Materials and Energy, 19:179-183, may 2019. doi:10.1016/j.nme.2019.02.012. ${ }^{20}$ Dirk Nicolai, Vadim Borsuk, Philipp Drews, Olaf Grulke, Klaus-Peter Hollfeld, Thorsten Krings, Yunfeng Liang, Christian Linsmeier, Olaf Neubauer, Guruparan Satheeswaran, Bernd Schweer, and Guido Offermanns. A multi-purpose manipulator system for W7-X as user facility for plasma edge investigation. Fusion Engineering and Design, 123:960-964, nov 2017. doi:10.1016/j.fusengdes.2017.03.013.
${ }^{21}$ G. Satheeswaran, K.P. Hollfeld, P. Drews, D. Nicolai, O. Neubauer, B. Schweer, and O. Grulke. A PCS7-based control and safety system for operation of the W7-X multi-purpose manipulator facility. Fusion Engineering and Design, 123:699-702, nov 2017. doi:10.1016/j.fusengdes.2017.05.125.

${ }^{22}$ Thomas W. Fredian and Joshua A. Stillerman. Mdsplus. current developments and future directions. Fusion Engineering and Design, 60(3):229 - 233, 2002. ISSN 09203796. doi:https://doi.org/10.1016/S09203796(02)00013-3. URL http: //www.sciencedirect.com/science/ article/pii/S0920379602000133. ${ }^{23}$ T. Fredian, J. Stillerman, and G. Manduchi. Mdsplus extensions for long pulse experiments. Fusion Engineering and Design, 83(2):317 - 320, 2008. ISSN 0920-3796. doi:

https://doi.org/10.1016/j.fusengdes.2007.08.022. URL http://www.sciencedirect. com/science/article/pii/

S0920379607004267. Proceedings of the 6th IAEA Technical Meeting on Control, Data Acquisition, and Remote Participation for Fusion Research.

${ }^{24}$ Samuel A. Lazerson, Yu Gao, Kenneth Hammond, Carsten Killer, Georg Schlisio, Matthias Otte, Christoph Biedermann, Monica Spolaore, Sergey Bozhenkov, Joachim Geiger, Olaf Grulke, Dirk Nicolai, Guruparan Satheeswaran, Holger 
Niemann, Marcin Jakubowski, Peter Brunner, Boyd Blackwell, Michael Endler, Drewelow, Aleix Puig Sitjes, Adnan Soren Klose, and Lukas Rudischhauser Ali, Barbara Cannas, Fabio Pisano, Ralph and. Tuning of the rotational transKönig, Glen Wurden, Gabor Kocsis, Tamás form in Wendelstein 7-X. Nuclear Szepesi, Uwe Wenzel, Matthias Mulsow, Fusion, 59(12):126004, sep 2019. doi: Kian Rahbarnia, Jonathan Schilling, 10.1088/1741-4326/ab3df0. URL https: Ulrich Neuner, Tamara Andreeva, Hen- //doi.org/10.1088/1741-4326/ab3df0. ning Thomsen, Jens Knauer, Kai Jakob 


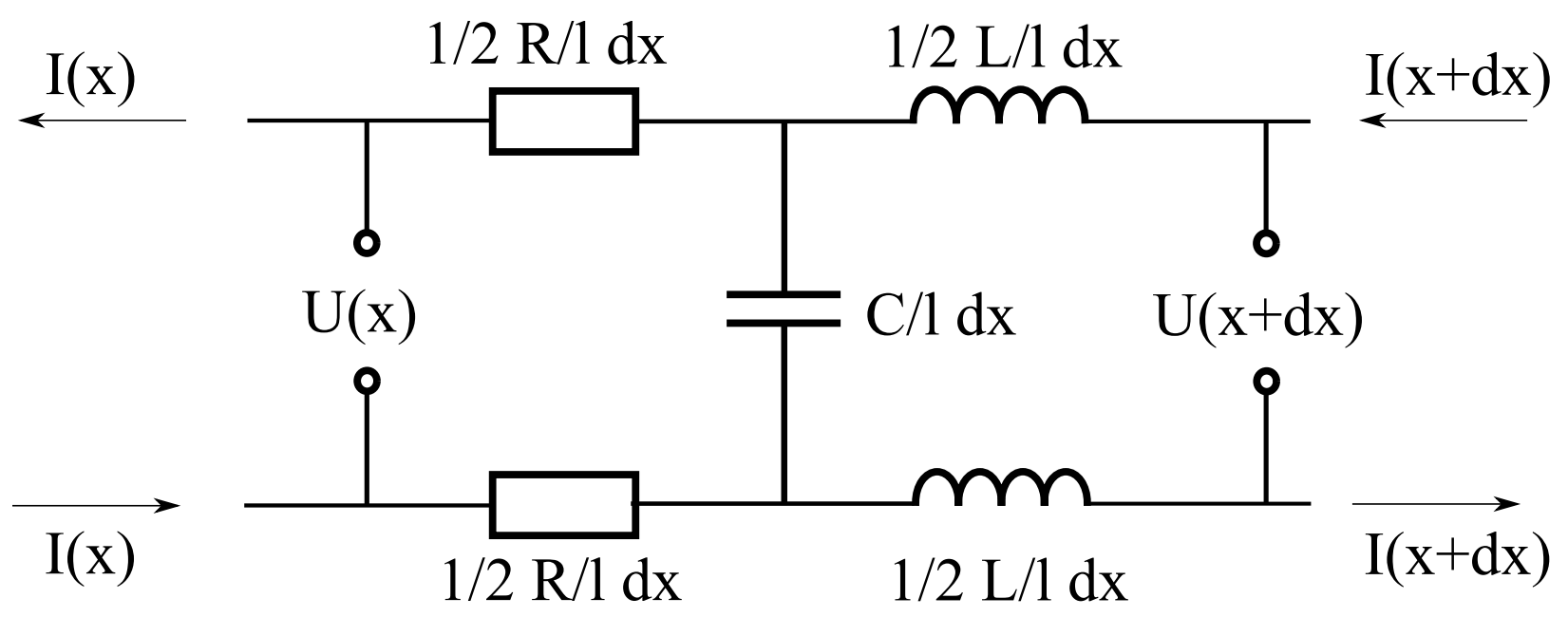

Figure 9. Schematic of the continuous cable model

\section{Appendix A: Derivation of the full transfer model}

Assuming the twisted wire pair to follow a standard differential LCR model, the differential signal propagation equations can be extracted from figure 9 as:

$$
\begin{aligned}
& \frac{d U}{d x}=-\frac{R_{\text {cable }}}{l} I(x)-\frac{L_{\text {cable }}}{l} \frac{d}{d t} I(x) \\
& \frac{d I}{d x}=-\frac{C_{\text {cable }}}{l} \frac{d}{d t} U(x)
\end{aligned}
$$

Using a propagating wave Ansatz $U, I(x, t)=U, I_{ \pm} \exp (i(\omega t \pm k x))$ yields the wave dispersion relation

$$
\begin{aligned}
\pm i k U_{ \pm} & =-\frac{R_{\text {cable }}}{l} I_{ \pm}-i \omega \frac{L_{\text {cable }}}{l} I_{ \pm} \\
\pm i k I_{ \pm} & =-i \omega \frac{C_{\text {cable }}}{l} U_{ \pm} \\
& \Rightarrow \\
-k^{2} & =i \omega \frac{R_{\text {cable }}}{l} \frac{C_{\text {cable }}}{l}-\omega^{2} \frac{L_{\text {cable }}}{l} \frac{C_{\text {cable }}}{l} \\
& \Rightarrow \\
k(\omega) & =\sqrt{\omega^{2} \frac{L_{\text {cable }} C_{\text {cable }}}{l^{2}}-i \omega \frac{R_{\text {cable }} C_{\text {cable }}}{l^{2}}}
\end{aligned}
$$


Assuming that the coil is connected at $x=0$ and the integrator input at $x=l$, one gets:

$$
\begin{aligned}
\tilde{U}_{\text {coil }} & =U_{+}+U_{-} \\
\tilde{U}_{\text {input }} & =U_{+} \exp (i k l)+U_{-} \exp (-i k l) \\
\tilde{I}_{\text {coil }} & =\frac{\omega}{k} \frac{C_{\text {cable }}}{l}\left(U_{-}-U_{+}\right) \\
& =Z(\omega)^{-1}\left(U_{-}-U_{+}\right) \\
\tilde{I}_{\text {input }} & =Z(\omega)^{-1}\left(\exp (-i k l) U_{-}-\exp (i k l) U_{+}\right)
\end{aligned}
$$

with the frequency-dependent cable impedance

$$
\begin{aligned}
Z(\omega) & =\left(\frac{\omega}{k} \frac{C_{\text {cable }}}{l}\right)^{-1} \\
& =\sqrt{\frac{L_{\text {cable }}}{C_{\text {cable }}}-\frac{i}{\omega} \frac{R_{\text {cable }}}{C_{\text {cable }}}}
\end{aligned}
$$

The coil follows a linear differential equation and can be subjected to the same rotating wave Ansatz

$$
\begin{aligned}
V_{\text {coil }} & =\frac{d}{d t}\left[A_{\text {coil }} B-L_{\text {coil }} I_{\text {coil }}\right]-R_{\text {coil }} I_{\text {coil }} \\
\tilde{V}_{\text {coil }} & =i \omega\left(A_{\text {coil }} \tilde{B}-L_{\text {coil }} \tilde{I}_{\text {coil }}\right)-R_{\text {coil }} \tilde{I}_{\text {coil }} \\
& =i \omega A_{\text {coil }} \tilde{B}-\left(R_{\text {coil }}+i \omega L_{\text {coil }}\right) \tilde{I}_{\text {coil }}
\end{aligned}
$$

while the integrator has a fixed input impedance of $R_{\text {meas }}=10 \mathrm{k} \Omega$, along with an optional capacitor $C_{\text {meas }}$ used in characterization to determine the coil inductance.

$$
\tilde{I}_{\text {input }}=\left(R_{\text {meas }}^{-1}+i \omega C_{\text {meas }}\right) \tilde{U}_{\text {input }}
$$

Expanding for $U_{ \pm}$yields:

$$
\begin{gathered}
U_{+}+U_{-}=i \omega A_{\text {coil }} \tilde{B}-\left(R_{\text {coil }}+i \omega L_{\text {coil }}\right) \frac{U_{-}-U_{+}}{Z} \\
\frac{\exp (-i k l) U_{-}-\exp (i k l) U_{+}}{Z}=\left(R_{\text {meas }}^{-1}+i \omega C_{\text {meas }}\right)\left[U_{+} \exp (i k l)+U_{-} \exp (-i k l)\right] \\
U_{-}=\frac{1+Z\left(R_{\text {meas }}^{-1}+i \omega C_{\text {meas }}\right)}{1-Z\left(R_{\text {meas }}^{-1}+i \omega C_{\text {meas }}\right)} \exp (2 i k l) U_{+}
\end{gathered}
$$

Let $\kappa=Z\left(R_{\text {meas }}^{-1}+i \omega C_{\text {meas }}\right)$ and $\lambda=Z^{-1}\left(R_{\text {coil }}+i \omega L_{\text {coil }}\right)$ be the normalized dimensionless output- and input-impedances. Then 


$$
\begin{aligned}
{\left[1+\frac{1+\kappa}{1-\kappa} \exp (2 i k l)\right] U_{+} } & =i \omega A_{\text {coil }} \tilde{B}+\lambda\left[1-\frac{1+\kappa}{1-\kappa}\right] U_{+} \\
& \Rightarrow \\
{\left[1-\lambda+\frac{1+\kappa}{1-\kappa}(\lambda+\exp (2 i k l))\right] U_{+} } & =i \omega A_{\text {coil }} \tilde{B} \\
U_{+} & =\frac{1}{1-\lambda+\frac{1+\kappa}{1-\kappa}(\lambda+\exp (2 i k l))} i \omega A \tilde{B} \\
U_{-} & =\frac{\frac{1+\kappa}{1-\kappa} \exp (2 i k l)}{1-\lambda+\frac{1+\kappa}{1-\kappa}(\lambda+\exp (2 i k l))} i \omega A \tilde{B}
\end{aligned}
$$

This then gives an input voltage on the integrator of

$$
\begin{aligned}
\tilde{U}_{\text {input }} & =\frac{\left(1+\frac{1+\kappa}{1-\kappa}\right) \exp (i k l)}{1-\lambda+\frac{1+\kappa}{1-\kappa}(\lambda+\exp (2 i k l))} i \omega A_{\text {coil }} \tilde{B} \\
& =\frac{2 \exp (i k l)}{(1-\lambda)(1-\kappa)+(1+\kappa)(\lambda+\exp (2 i k l))} i \omega A_{\text {coil }} \tilde{B}
\end{aligned}
$$

In the case of $k l \approx 0$ (neglecting forward and backward phase delays due to the cable), one can simplify this expression to:

$$
\begin{aligned}
\tilde{U}_{\text {input }} & \approx \frac{2}{(1-\lambda)(1-\kappa)+(1+\kappa)(\lambda+1)} i \omega A_{\text {coil }} \tilde{B} \\
& =\frac{i \omega A_{\text {coil }} \tilde{B}}{1+\lambda \kappa} \\
& =\frac{i \omega A_{\text {coil }} \tilde{B}}{1+\frac{R_{\text {coil }}}{R_{\text {meas }}}+i \omega\left(R_{\text {coil }} C_{\text {meas }}+\frac{L_{\text {coil }}}{R_{\text {meas }}}\right)-\omega^{2} L_{\text {coil }} C_{\text {meas }}}
\end{aligned}
$$

